\title{
Etude de la variabilité agronomique et biochimique des quatre génotypes d'une variété de blé dur cultivé en Algérie (Triticum durum Desf.)
}

\author{
Chehili Fatima, Doctorante \\ Boudour Leila, Professeur \\ Bouchtab Karima, Maitre-assistante
}

Département de Biologie et Ecologie Végétale, Faculté des Sciences de la

Nature et de la Vie, Université Frères Mentouri Constantine 1, Algérie

doi: 10.19044/esj.2017.v13n9p409 $\quad$ URL:http://dx.doi.org/10.19044/esj.2017.v13n9p409

\begin{abstract}
The present study deals with four genotypes belonging to the Algerian durum wheat melanopus variety. In the experiment carried out in the greenhouse at Mentouri Constantine1Chaâbat Ersas University, it is measured the morpho-physiological parameters (stem height, cervical length, ear, barbs, leaf area, relative content Water yield and chlorophyll); the yield parameters (number of grains, number of spikelets per spike and weight of one thousand grains) and the biochemical parameters (total protein).

From the results of the morphological parameters, it appears that the genotypes differ essentially by the leaf area. For the physiological parameters: total chlorophyll content and relative water content, no variation between the four genotypes was observed. As for the biochemical study evaluated by the total proteins, this made it possible to better differentiate the 4 genotypes and showed a remarkable intra-varietal polymorphism.
\end{abstract}

Keywords: Durum Wheat, Genotypes, Morphology, Physiology, Proteins And Polymorphism.

\section{Résumé}

La présente étude porte sur 4 génotypes appartenant à la variété melanopus de blé dur algérien. Dans l'expérimentation menée en serre à Chaâbat Ersas Université des frères Mentouri Constantine1, sont mesurés des paramètres morpho-physiologiques (hauteur de la tige, longueurs du col, de l'épi, et des barbes, surface foliaire, teneur relative en eau et taux de chlorophylle), du rendement (nombre de grains, nombre d'épillets par épi et poids des mille grains) et biochimique (protéines totales). 
A partir des résultats des paramètres morphologiques, il apparait que les génotypes différencier essentiellement par la surface foliaire. Pour les paramètres physiologiques: teneur en chlorophylle totale et teneur relative en eau, aucune variation entre les quatre génotypes n'a été observée. Quant à l'étude biochimique évaluée par les protéines totales, celle-ci a permis de mieux différencier les 4 génotypes et a montré un polymorphisme intravariétal remarquable.

Mots clés: Blé Dur, Génotypes, Morphologie, Physiologie, Protéines Et Polymorphisme.

\section{Introduction}

Le blé est actuellement l'une des céréales la plus cultivée au monde avec une production annuelle totale de prés de 680 millions de tonnes (Mariana, 2011). En effet, la presque totalité de la nutrition de la population mondiale est fournie par les aliments en grains dont $95 \%$ sont produits par les cultures céréalières.

En Algérie, le blé dur (Triticum durum Desf.) est la première céréale cultivée dans le pays et occupe annuellement plus d'un million d'hectares. Cependant, sa production est encore faible, elle ne couvre que 20 à $25 \%$ des besoins du pays, le reste étant importé (Anonyme1, 2008). La cause principale de la faiblesse de la production du blé dur soit 15,4 quintaux/hectare (ONFAA, 2015), est liée à des contraintes abiotiques (pluviométrie surtout), biotiques (adventices) et humaines (itinéraires techniques appliqués etc...) (Chellali, 2007).

Selon Roudart, (2006) le blé dur est l'une des principales ressources alimentaires de l'humanité et des animaux à raison de $75 \%$ et $15 \%$ de la production respectivement, et le reste à des usages non alimentaires (Feillet, 2004). La semoule issue des grains de blé dur est à l'origine de produits très divers: pâtes alimentaires, couscous et bien d'autres produits comme le pain, le frik et divers gâteaux (Troccoli et al., 2000). La paille est également utilisée comme litière et comme aliment pour les animaux. Le blé dur a une grande valeur nutritionnelle, suite à sa richesse en protéines et à la présence du gluten qui a la propriété unique de former après hydratation une masse cohérente (Amri, 2010). Il renferme en plus des acides aminés, des lipides, des glucides, quelques sels minéraux et des vitamines.

Chez le blé, plusieurs caractères adaptatifs d'ordre phénologique, morphologique et physiologique, participent à l'amélioration de la tolérance aux stress abiotiques (Boudour, 2006). L'étude de ces paramètres peut se réaliser par différents outils d'analyse, les uns reposent sur des critères morpho-physiologiques simples et fiables, les autres récents et plus performants font appel à des marqueurs biochimique et moléculaire. 
Dans ce travail, une étude combinée entre le potentiel de production et d'adaptation aux conditions environnementales semi-arides de la région de Constantine est réalisée afin d'évaluer la variabilité morpho-physiologique, rendement et biochimique pouvant exister entre des génotypes d'une variété de blé dur cultivé en Algérie.

\section{Matériel et méthodes}

\section{Matériel végétal}

Le matériel végétal utilisé dans cette étude est constitué de quatre génotypes de la variété melanopus de blé dur (Triticum durum Desf.) algérien prélevés de la collection étudiée par Boudour, 2004-2006 caractérisée par épi roux, glume glabre rouge, paille creuse à demi creuse, grain rouge et compact.

\section{Conditions de culture}

La germination des graines est réalisée dans des boites de pétri à raison de 10 graines/ boite. Les plantules obtenues sont mises dans des pots de $5 \mathrm{Kg}$ installés dans une serre à Chaâbat Ersas Université des frères Mentouri, Constantine1.

\section{Paramètres mesurés}

\section{Paramètres morphologiques}

Les paramètres morphologiques mesurés sont :

- la hauteur de la plante (HP) exprimée en $\mathrm{cm}$,

- la longueur du col (LC) exprimée en $\mathrm{cm}$ mesurée à la maturité à partir du dernier nœud,

- la longueur de l'épi (LE) exprimée en cm,

- la longueur des barbes (LB) exprimée en cm mesurée au stade maturité à partir du $1 / 3$ de

l'épi jusqu'au sommet des barbes,

- la surface foliaire (SF) exprimée en $\mathrm{cm}^{2}$ est mesurée à l'aide d'un appareil« Digital planimètre $»$.

Pour chaque paramètre 4 répétitions sont effectuées sur différentes plantes.

\section{Paramètres physiologiques}

- la teneur relative en eau (TRE): est mesurée selon la méthode décrite par Barrs, (1968) :

PF: poids fr:

$$
\operatorname{TRE}(\%)=[(\mathbf{P F}-\mathrm{PS}) /(\mathrm{PT}-\mathrm{PS})] \times 100
$$

échantillons foliaires exprimés en $\mathrm{mg}$. 


\section{- la chlorophylle totale}

Le taux de chlorophylle au niveau des feuilles est mesuré à l'aide d'une chlorophylle - mètre SPAD 502 de Minolta.

\section{Technique d'analyse}

La technique utilisée est l'électrophorèse mono ( SDS- PAGE) proposée par Laemmli, (1970) et modifiée par Payne et al., (1979). La séparation est faite sur gel vertical discontinu, en présence d'un détergent ionisé (Sodium Dodocyl Sulfate SDS). Cette méthode facile, reproductible et rapide permet de mesurer, comparer et caractériser les protéines. Elle comprend les étapes suivantes :

\section{Extraction des protéines totales}

Quatre graines de blé sont broyées en présence d'azote liquide à -198 ${ }^{\circ} \mathrm{C}, 0.4 \mathrm{~g}$ de farine sont homogénéisés dans $350 \mu \mathrm{l}$ (2x sample tampon). Le mélange recueilli est conservé dans des Eppendorfs à - $4{ }^{\circ} \mathrm{c}$ pendant une nuit. Le tout est homogénéisé à l'aide d'un vortex pendant 5 secondes. Après centrifugation $\left(15 \mathrm{mn}\right.$ à $4^{\circ} \mathrm{C}$ à $\left.12.000 \mathrm{tr} / \mathrm{min}\right)$, le surnageant riche en protéines totales est récupéré et conservé à $-20^{\circ} \mathrm{c}$ jusqu’à utilisation.

\section{- Préparation d'échantillon}

L'analyse électrophorétique est faite avec un volume de $35 \mu \mathrm{l}$ de l'extrait de protéine, auquel on ajoute le même volume de SDS ( 2x solution tampon $\mathrm{pH}=6,8$ ), plus $7 \mu \mathrm{l}$ Mercaptoethanol et $7 \mu \mathrm{l}$ bromophenol bleu. Le mélange est porté à ébullition dans un bain-marie à $100^{\circ} \mathrm{C}$ durant $5 \mathrm{~min}$, puis homogénéisé pendant 5-10 secondes et centrifugé durant 1 minute.

- Préparation des gels

Le support d'électrophorèse est formé d'un mélange de gels : gel de séparation à $15 \%$ et $\mathrm{pH}=8,8$ et un gel de concentration à $5 \%$ et un $\mathrm{pH}$ $=6,8$.

\section{- Coloration et décoloration}

Les gels sont retirés des plaques et les protéines sont fixées dans une solution colorée au bleu de coomassie (0.1 bleu de coomassis R-250, 45ml Méthanol, $10 \mathrm{ml}$ Acide Acétique, $45 \mathrm{ml} \mathrm{H}_{2} \mathrm{O}$ ) pendant une nuit. Les gels sont décolorés pendant 24 heures par une solution de $20 \mathrm{ml}$ méthanol : $80 \mathrm{ml}$ $\mathrm{H}_{2} \mathrm{O}$ avec une agitation vigoureuse, ensuite lavés plusieurs fois avec l'eau distillée. Les gels sont photographiés par le système de détection d'image Gel Doc-print II.

\section{Analyse statistique}

Les résultats obtenus des différents paramètres pour les quatre génotypes de la variété mélanopus sont interprétés grâce aux analyses statistiques multi variées telle que l'analyse de la variance (ANOVA) et l'analyse en composantes principales (ACP) en utilisant le logiciel statistica, 
(2008). De plus nous avons fait appel à la matrice de similarité et le dendrogramme à l'aide du logiciel statistica version 6 .

\section{Résultats et discussion}

\section{Paramètres morphologiques}

- hauteur de la tige, longueur du col, longueur de l'épi, longueur des barbes

Ces quatre paramètres morphologiques représentés dans la figure 1 montrent que :

- la hauteur de la tige (HT) varie entre 42,16 et $46,5 \mathrm{~cm}$. Chez les génotypes G3 et G4 sont observées les valeurs les plus élevées de 46,5 et $45 \mathrm{~cm}$ respectivement, alors que les valeurs les plus faibles sont enregistrées chez les génotypes G1et G2 respectivement de 43,5 et 42,16cm.

- la longueur du col (LC) des quatre génotypes étudiés se situe entre 11,33 et $15,5 \mathrm{~cm}$. Les valeurs les plus élevées sont attribués aux génotypes G2 et G3 de 13,83 et $15.5 \mathrm{~cm}$ respectivement, d'autre part les génotypes G1, G4 se caractérisent par une petite taille de 9,16 et $11,33 \mathrm{~cm}$ respectivement.

- la longueur de l'épi (LE) des quatre génotypes varie entre 3 et $4 \mathrm{~cm}$, comme pour le paramètre précédent.

- la longueur des barbes (LB) est comprise entre $8,33 \mathrm{~cm}$ chez le génotype $\mathrm{G} 2$ et $10,33 \mathrm{~cm}$ chez le génotype G4.

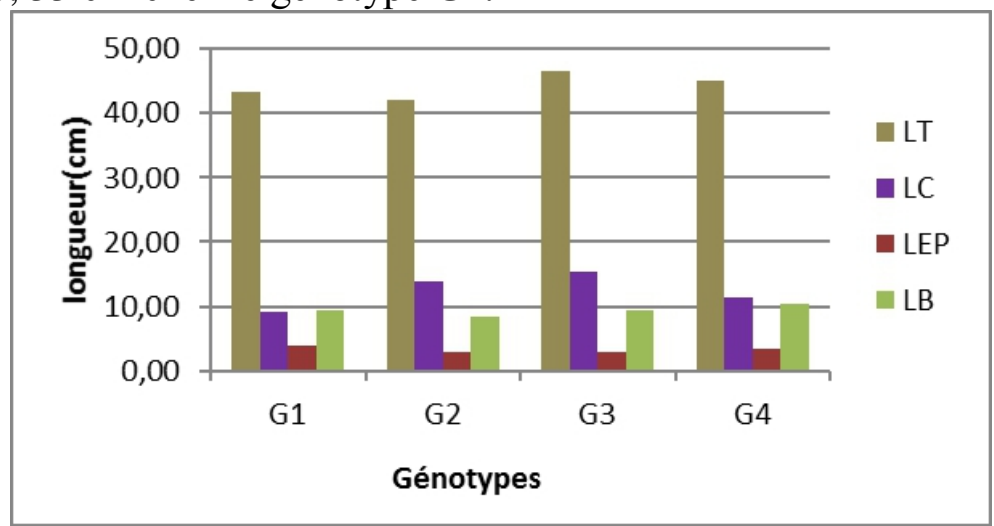

Fig. 1. Paramètres morphologiques (hauteur de la tige, longueur du col, longueur de l'épi, longueur des barbes) de la variété melanopus de blé dur algérien.

La hauteur de la tige est un caractère important car le taux d'accumulation de la matière sèche en pré anthèse, et la capacité de translocation des assimilâts stockés dans la tige jouent un rôle dans l'adaptation de la variété aux contraintes climatiques (Belkharchouche et al., 1989).

La longueur du col de l'épi est un caractère variétal souvent proposé comme critère de sélection de génotypes tolérants au déficit hydrique (Fisher et Maurer, 1978). En effet son rôle s'expliquerait par les quantités 
d'assimilats stockées dans cette partie de la plante qui sont susceptibles d'être transportées vers le grain en conditions de déficit hydrique terminal (Gate et al. ,1992). D’après Annich Chiarico, (1993) cité par Ben Mimoun, (1994) la longueur du col de l'épi est proportionnelle à la quantité d'eau absorbée durant le cycle de développement.

Concernant la longueur de l'épi qui est une caractéristique variétale peu influencée par les variations du milieu, elle est fonction de la quantité d'eau réservée durant le cycle végétatif (Anonyme2, 2008).

La longueur de la barbe chez le blé peut faire augmenter la possibilité d'utilisation de l'eau et l'élaboration de la matière sèche lors de la phase de maturation. Selon Blottière, (2003) et Nemmar, (1980) la longueur de la barbe contribue également à une limitation des pertes en eau. Ce paramètre morphologique semble étroitement lié à la tolérance au déficit hydrique terminal tout au moins chez le blé dur (Slama, 2005).

\section{- la surface foliaire (SF)}

La surface foliaire des quatre génotypes varie de $10,04 \mathrm{~cm}^{2}$ à $15,96 \mathrm{~cm}^{2}$. Les deux génotypes G1 et G3 possèdent les valeurs les plus élevées de 15, 95 et $15,96 \mathrm{~cm}^{2}$ respectivement, alors que les surfaces des deux génotypes $\mathrm{G} 2$ et G4 sont faibles respectivement de 12,27 et $10,04 \mathrm{~cm}^{2}$ (Fig.2).

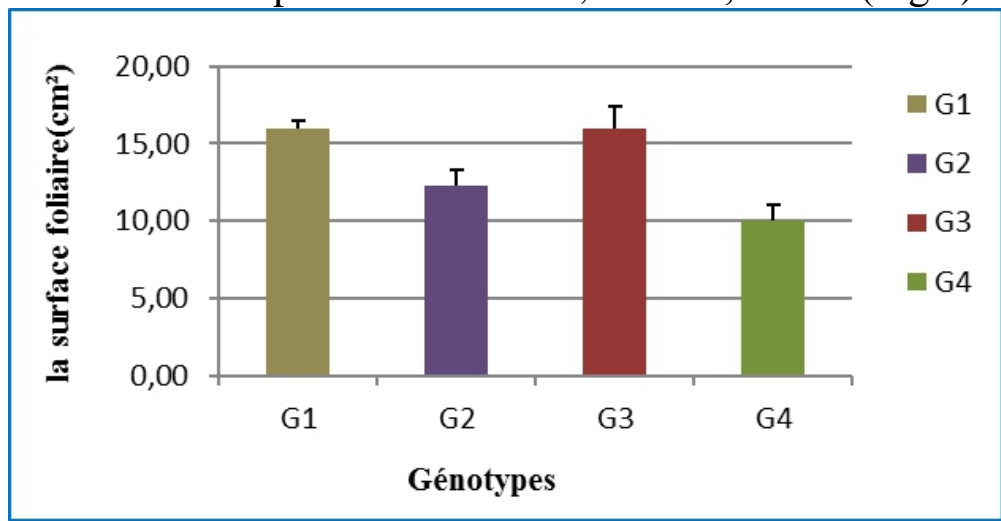

Fig. 2. Surface foliaire des quatre génotypes de la variété melanopus de blé dur algérien.

L'analyse de la variance au seuil 5\% montre une différence hautement significative entre les variétés étudiées. Le test $\mathrm{SNK}$ à $5 \%$ confirme ces résultats et repartit les variétés en trois groupes distincts:

- le premier groupe formé des génotypes G1et G3,

- le deuxième du génotype G2,

- le troisième du génotype G4. 
La surface foliaire est un déterminisme important de la transpiration, en effet, l'une des premières réactions des plantes au déficit hydrique est de réduire la surface foliaire (Lebon et al., 2004).

\section{Paramètres physiologiques \\ - la chlorophylle totale (Chl)}

Les valeurs moyennes obtenues se situent entre 25,47 et 28,80 unités SPAD pour les quatre génotypes de la variété melanopus. Chez les deux génotypes G3 et G4 sont observées les moyennes maximales de 27 et 28,80 unités SPAD respectivement. La valeur minimale de 25,47 unités SPAD est enregistrée chez le génotype G1 suivi par le génotype G2 dont la teneur est de 25,68 unités SPAD (fig. 3A).

\section{- la teneur relative en eau (TRE)}

Les variations de la teneur relative en eau des quatre génotypes G1, G2, G3 et G4 sont faibles. Elles varient de $76,09 \%$ chez le génotype G4 à $84.3 \%$ chez le génotype G2 (Fig. 3B).
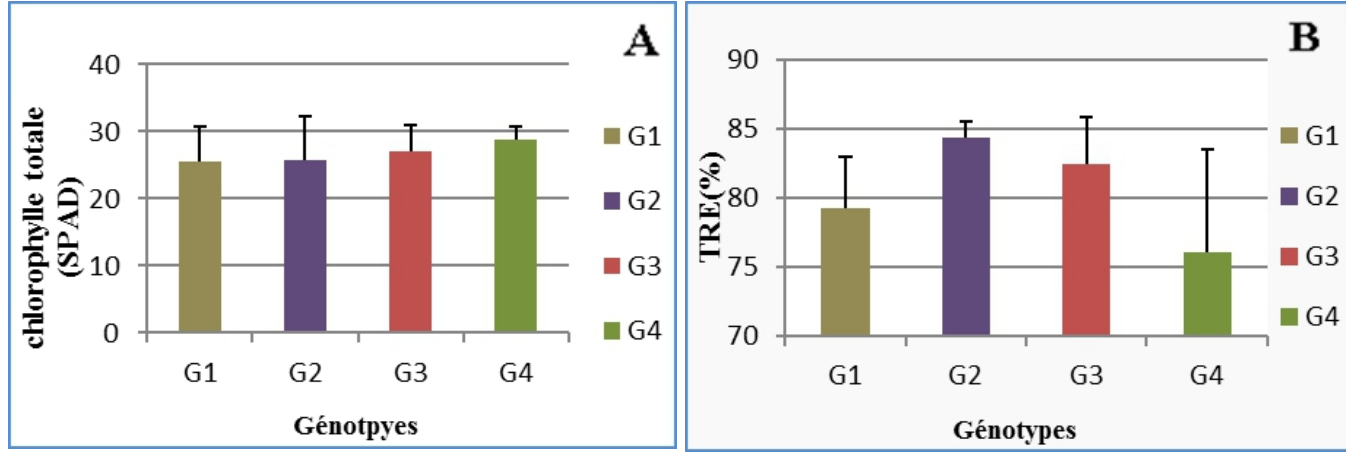

Fig. 3. Paramètres physiologiques des quatre génotypes de la variété melanopus de blé dur algérien, A: Chlorophylle totale, B: Teneur relative en eau.

L'analyse de la variance (ANOVA) des paramètres physiologiques (Chlorophyle totale et teneur relative en eau) n'indique aucune différence significative au seuil de 5\% entre les quatre génotypes. Par ailleurs le test de comparaisons multiples SNK au seuil de 5\% les classe en un seul groupe homogène.

Ces génotypes montrent une faible teneur en chlorophylle comparativement à celle obtenue dans les mêmes génotypes cultivés en plein champ. Ceci peut- être dû aux contraintes abiotiques qui existent dans la serre. En effet, les paramètres environnementaux tels que la lumière, la température et la disponibilité en eau influencent la teneur en chlorophylle des feuilles, en plus d'autres facteurs comme l'âge et la position des feuilles (Hikosaka et al., 2006).

La TRE dans les feuilles est un bon indicateur de l'état hydrique de la plante, utilisée surtout pour évaluer la tolérance au stress hydrique et salin. 
Le manque d'eau est un élément déterminant pour la croissance des plantes, particulièrement en région aride et semi aride (Albouchi et al., 2000).

\section{Composantes du rendement}

\section{- le nombre d'épillets par épi (NE/E)}

Le nombre d'épillets par épi des quatre génotypes étudiés est compris entre 12,33 et 15,33 épillets par épi. Les génotypes G3 et G4 enregistrent le nombre le plus élevé de l'ordre de 15,33 et 15 épillets respectivement. Les G1 et G2 ont un faible nombre d'épillets par épi avec des valeurs de 14 et 12,33 épillets par épi respectivement (Fig.4 A).

\section{- le poids de mille grains (PMG)}

Le poids de mille grains des quatre génotypes étudiés varie de 56 à 42g. , les génotypes G1 et G 3 enregistrent le poids le plus élevé de l'ordre de 56 g. alors que les génotypes G2 et G4 ont un poids de mille grains réduit de 45 et $42 \mathrm{~g}$ respectivement (Fig. 4B).

\section{- le nombre de grains par épi (NG/E)}

Les génotypes G1 et G4 montrent les valeurs les plus élevées de 11,33et 10 grains par épi respectivement. Tandis que, les génotypes G2 et G3 présentent les plus faibles valeurs de 7,33 et 9,66 grains par épi respectivement (Fig. $4 \mathrm{C}$ ).
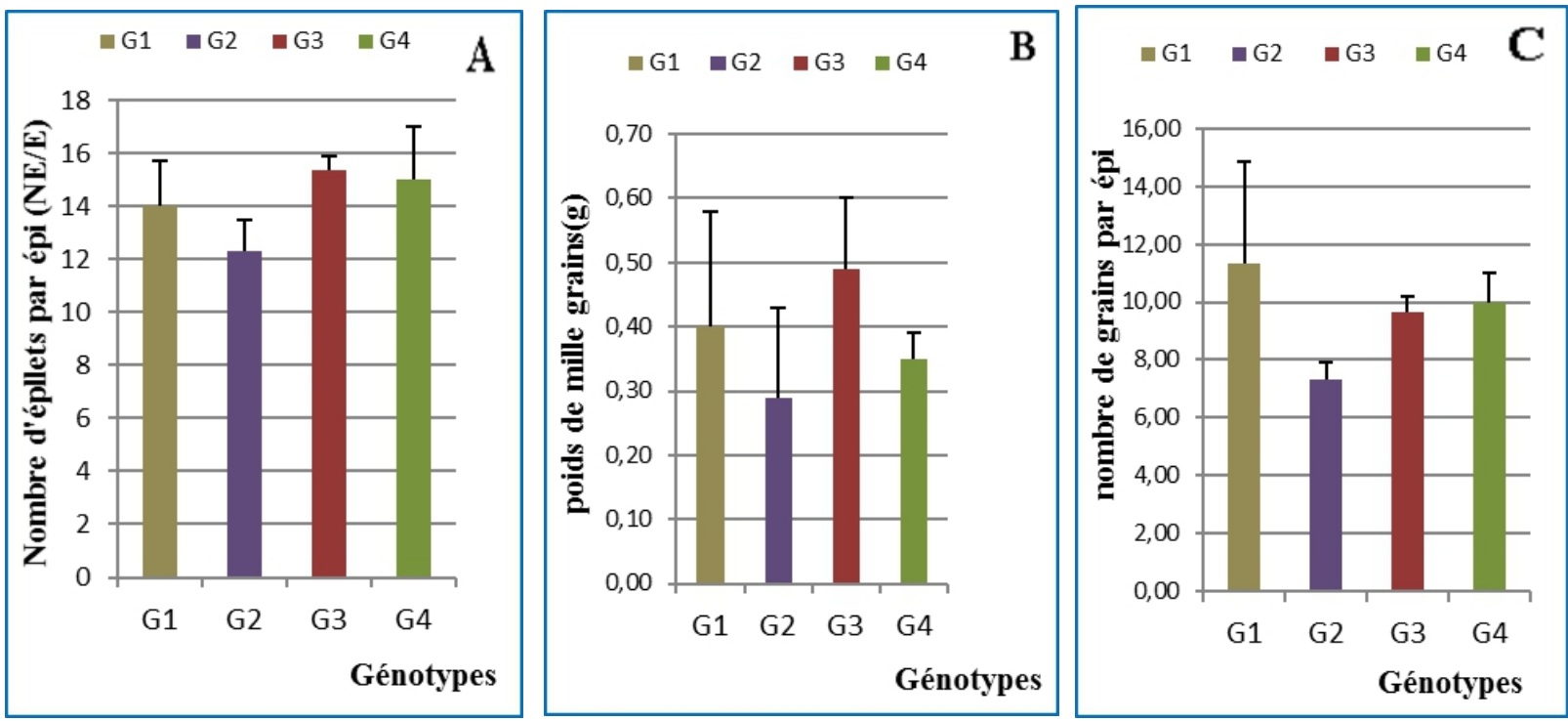

Fig.4. Composantes du rendement des quatre génotypes de la variété melanopus de blé dur algérien.

A: Nombre d'épillets par épi, B : Poids de mille grains, C : Nombre des grains par épi des 4 génotypes

D'après l'ANOVA, les génotypes étudiés ne présentent aucune différence significative, pour les deux composantes du rendement (nombre d'épillets par épi et poids de mille grains). Aussi le test SNK à 5\% confirme 
ces résultats et les classe en un seul groupe homogène. Alors que l'analyse de la variance montre une différence significative entre les quatre génotypes de la variété pour le paramètre nombre des grains par épi. Le test SNK le confirme et sépare le génotype G1, le plus performant du reste des génotypes qui forment un seul groupe.

Le poids de l'épi au stade épiaison est un bon indicateur du rendement potentiel à espérer lorsque le remplissage du grain se fait dans des conditions de croissance assez favorables (Belkherchouche et al., 2009).

Le rendement en grains du blé est plus lié à la fertilité de l'épi qu'au poids moyen du grain, ceci ne veut nullement dire que le nombre d'épis et le poids de 1000 grains ne jouent aucun rôle sous climat variable (Erchidi et al., 2000).

\section{Analyse de variabilité morpho-physiologique et du rendement}

Une analyse en composantes principales (ACP) effectuée sur les quatre génotypes de la variété melanopus en considérant l'ensemble des paramètres morpho-physiologiques et du rendement a montré que l'information fournie par les axes 1, 2 et 3 sont de 40,53\%, 35,16\% et 24,31\% respectivement. Pour cela, l'étude est réalisée dans les deux plans $1 / 2$ et $1 / 3$.

\section{Matrice des corrélations entre les variables}

L'analyse des corrélations entre les paramètres mesurés montre qu'il y a des liaisons positives et négatives entre ces paramètres variant d'un faible à une forte corrélation $\left(\mathrm{Tab}^{\circ} 1\right)$. Les liens les plus importants sont :

- la longueur de la tige (HP) fortement et positivement corrélée au nombre d'épillets par épi $(\mathrm{NE} / \mathrm{E})$ avec $\mathrm{r}=0,94$, moyennement et positivement aux variables: longueur des barbes (LB) avec $r=0.65$ et taux de chlorophylle(Chlo) avec $\mathrm{r}=0,63$.

- la longueur de col (LC) d'une part positivement et moyennement corrélée à la variable

teneur relative en eau (TRE) $(\mathrm{r}=0,66)$ et d'autre part négativement très liée à la longueur de l'épi (LE) $\mathrm{r}=0.96$ et moyennement au nombre de grains par épi $(\mathrm{NE} / \mathrm{E}) \mathrm{r}=0,65$.

- la longueur de l'épi (LE) fortement et positivement corrélée au nombre des grains par épi $(\mathrm{NG} / \mathrm{E})(\mathrm{r}=0,82)$.

- la longueur des barbes (LB) fortement et positivement liée aux longueurs des barbes (LB) $(\mathrm{r}=0,85)$, au taux de chlorophylle totale (Chlo) $(\mathrm{r}=0,77)$ et au nombre des grains par épi $(\mathrm{NG} / \mathrm{E})(\mathrm{r}=0,72)$. Aussi elle est négativement très corrélée à la variable teneur relative en eau (TRE) $\mathrm{r}=0,90$. 
Tableau $\mathbf{n}^{\circ} 1$ : Matrice des coefficients de corrélation des différentes variables analysées.

\begin{tabular}{|lllllllllll|}
\hline & LT & LC & LEP & LB & SF & Chlo & TRE & NE & NGE & PMG \\
\hline HT & 1 & & & & & & & & & \\
LC & 0,388 & 1 & & & & & & & & \\
LEP & $-0,198$ & $-0,964$ & 1 & & & & & & & \\
LB & 0,654 & $-0,370$ & 0,459 & 1 & & & & & & \\
SF & 0,199 & 0,065 & 0,154 & $-0,192$ & 1 & & & & & \\
Chlo & 0,633 & 0,099 & 0,112 & 0,778 & $-0,614$ & 1 & & & & \\
TRE & $-0,281$ & 0,667 & 0,670 & $-0,909$ & 0,384 & $-0,661$ & 1 & & & \\
NE & 0,941 & 0,056 & 0,130 & 0,854 & 0,159 & 0,675 & $-0,563$ & 1 & & \\
NGE & 0,378 & $-0,653$ & 0,820 & 0,726 & 0,402 & 0,135 & $-0,690$ & 0,637 & 1 & \\
PMG & 0,298 & 0,010 & 0,226 & $-0,048$ & 0,990 & $-0,511$ & 0,256 & 0,287 & 0,516 & 0,793 \\
\hline
\end{tabular}

\section{Etude des variables}

Dans le plan $1 / 2$ (fig.5A), l'axe 1 est formé du coté positif principalement par le nombre d'épillets par épi (NE/E), la longueur des barbes (LB), le nombre des grains par épi (NG / E), la hauteur de la tige (HT) et du coté négatif par la teneur relative en eau (TRE), donc l'axe 1 pourrait être défini comme un axe morpho- physiologique et de rendement.

Les variables: surface foliaire (SF), longueur de col (LC), poids de mille grains $(\mathrm{PMG})$ déterminent l'axe 2 du coté négatif. Cet axe peut être qualifié d'axe morphologique et de rendement.

Dans le plan 1/3 (fig.5B), on peut voir que le taux de chlorophylle totale (Chlo) définit l'axe 3 du coté positif et la longueur de l'épi(LEP) du coté négatif.

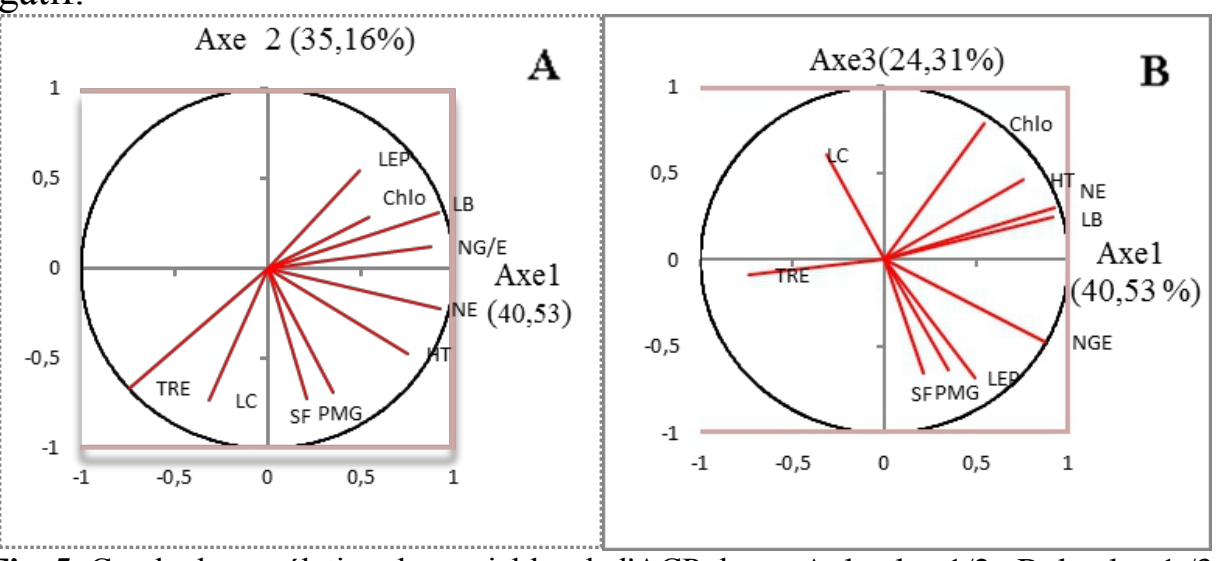

Fig. 5. Cercle de corrélation des variables de l'ACP dans : A le plan 1/2, B le plan 1/3. Etude des individus 
La représentation graphique des individus dans le plan 1/2 (Fig. 7A) laisse apparaitre clairement que :

Les génotypes G1 et G4 sont très proches et se caractérisent par les mêmes variables à savoir nombre d'épillets par épi $(\mathrm{NE} / \mathrm{E})$, longueur des barbes (LB), nombre des grains par épi (NG / E), hauteur de la tige (HT). Ces derniers s'opposent d'une part au génotype G2 qui se distingue plutôt par une teneur relative en eau (TRE) élevée et d'autre part au génotype G3 du coté négatif de l'axe 2 comprenant une surface foliaire (SF), une longueur de col (LC) et un poids de mille grains (PMG) importants.

La dispersion des génotypes dans le plan 1/3 (Fig. 7B) montre que le G4 se distingue par un fort taux de chlorophylle totale (Chlo) du coté positif alors que le génotype G1 situé du coté négatif de l'axe 3 par une longueur de l'épi(LEP) élevée.

En conclusion, l'analyse en composantes principales révèle 3 groupes distincts qui se différencient par les paramètres morpho-physiologiques et du rendement :

- Le premier groupe constitué par les génotypes (G1et G4) qui se caractérisent par les mêmes paramètres mais se différencient par deux variables: longueur de l'épi(LEP) importante chez le G1et taux de chlorophylle totale (Chlo) élevé chez le génotype G4.

- Le deuxième groupe formé par le génotype (G3) s'oppose aux génotypes G1, G4.

- Le dernier groupe comprend le génotype (G2) tout à fait excentré vers le coté négatif de l'axel.

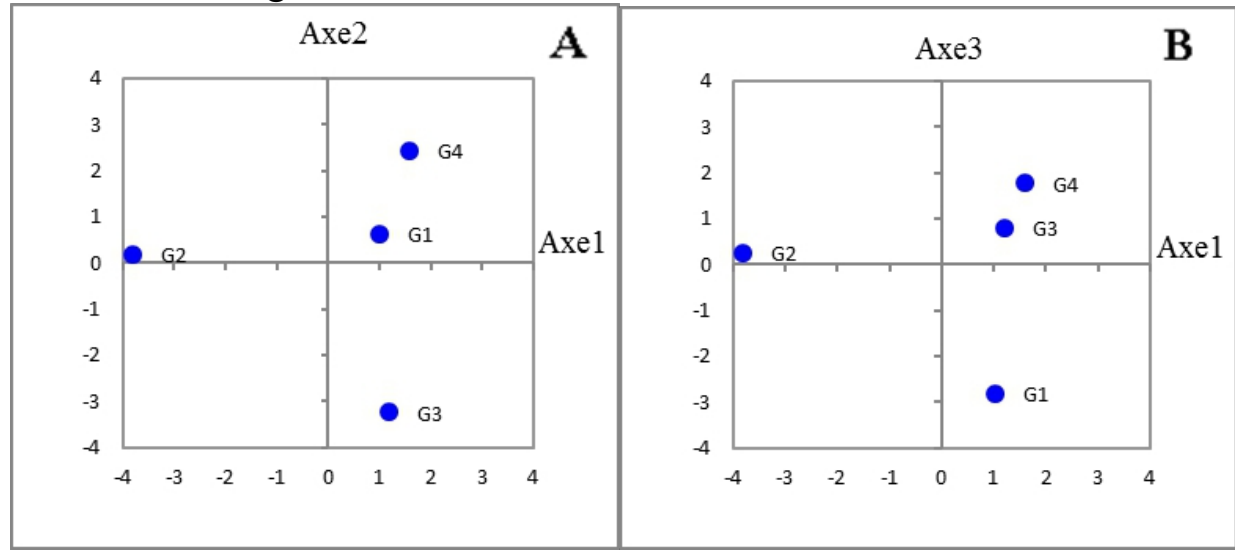

Fig. 6. Représentation graphique des quatre génotypes sur le plan $1 / 2$ et $1 / 3$ de l'ACP

\section{Paramètre biochimique : Les protéines totales}

L'analyse des protéines totales des grains des quatre génotypes de blé dur par la technique d'électrophorèse (SDS-PAGE) a révélé un certain polymorphisme (fig. 7). 
Cette analyse est basée sur le nombre, l'intensité et le poids moléculaire des bandes observées sur le gel (fig. 7).

Le nombre total des bandes est de 112 de poids moléculaire variant de 10 à $148 \mathrm{~K} \mathrm{Da}\left(\mathrm{Tab} \mathrm{n}^{\circ}\right.$ 3), les génotypes G1et G4 présentent 28 bandes avec un taux de polymorphisme de $57,14 \%$.

Les génotypes $\mathrm{G} 2$ et $\mathrm{G} 3$ possèdent respectivement 30 et 26 bandes et se caractérisent par un taux de polymorphisme égal de 60 et $53.84 \%$ respectivement $\left(\operatorname{Tab}^{\circ} 3\right)$.

D'après Jasso, 2002 l'augmentation de l'intensité des bandes est le résultat de l'augmentation des protéines, ce qui constitue un marqueur moléculaire important.

Fig. 7. Profil électrophorètique SDS-PAGE des protéines totales

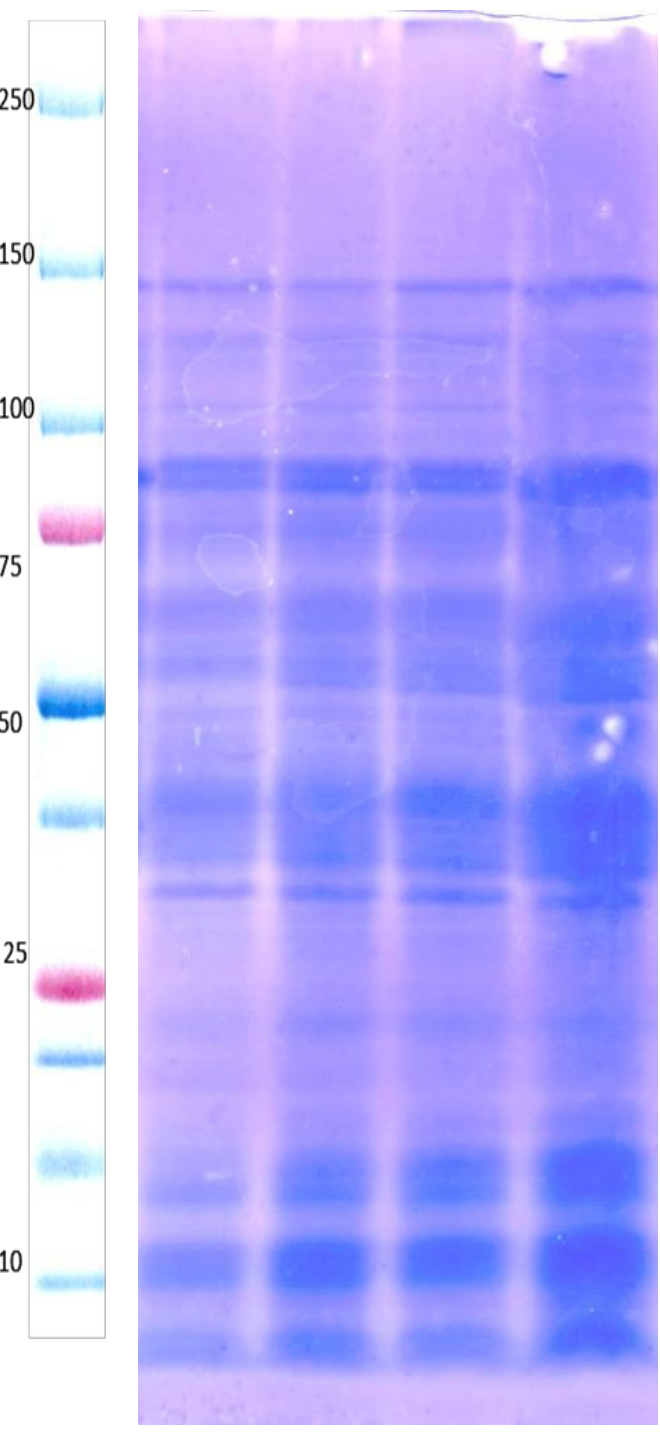

\begin{tabular}{|c|c|c|c|c|c|c|}
\hline \multirow[b]{2}{*}{$\mathrm{nb}$} & \multirow[b]{2}{*}{ PM } & \multicolumn{3}{|c|}{$\begin{array}{r}\text { Individus } \\
\text { (génotypes) }\end{array}$} & \multirow[b]{2}{*}{4} & \multirow[t]{2}{*}{ Bande } \\
\hline & & 1 & 2 & 3 & & \\
\hline 1 & 148 & + & + & + & + & $\mathrm{M}$ \\
\hline 2 & 130 & + & + & + & + & $\mathrm{M}$ \\
\hline 3 & 120 & + & + & + & + & $\mathrm{M}$ \\
\hline 4 & 110 & + & + & + & + & $\mathrm{M}$ \\
\hline 5 & 100 & + & + & + & + & $\mathrm{M}$ \\
\hline 6 & 95 & + & + & + & + & $\mathrm{M}$ \\
\hline 7 & 90 & + & - & - & - & $\mathrm{U}(+)$ \\
\hline 8 & 77 & - & + & + & - & $\mathrm{P}$ \\
\hline 9 & 75 & - & - & - & + & $\mathrm{U}(+)$ \\
\hline 10 & 74 & + & - & - & - & $\mathrm{U}(+)$ \\
\hline 11 & 72 & - & + & + & - & $\mathrm{P}$ \\
\hline 12 & 70 & - & + & + & + & $\mathrm{U}(-)$ \\
\hline 13 & 65 & + & + & - & + & $\mathrm{U}(-)$ \\
\hline 14 & 62 & + & - & - & - & $\mathrm{U}(+)$ \\
\hline 15 & 60 & - & + & - & + & $\mathrm{P}$ \\
\hline 16 & 59 & + & + & + & - & U(-) \\
\hline 17 & 54 & - & - & + & + & $\mathrm{P}$ \\
\hline 18 & 53 & + & + & - & - & $\mathrm{P}$ \\
\hline 19 & 50 & + & - & - & - & $\mathrm{U}(+)$ \\
\hline 20 & 48 & - & - & - & + & $\mathrm{U}(+)$ \\
\hline 21 & 47 & - & + & - & - & $\mathrm{U}(+)$ \\
\hline 22 & 45 & + & - & - & + & $\mathrm{P}$ \\
\hline 23 & 43 & - & + & + & - & $\mathrm{P}$ \\
\hline 24 & 41 & + & - & - & - & $\mathrm{U}(+)$ \\
\hline 25 & 40 & - & - & - & + & $\mathrm{U}(+)$ \\
\hline 26 & 39 & + & + & + & - & $\mathrm{U}(-)$ \\
\hline 27 & 38 & - & + & + & - & $\mathrm{P}$ \\
\hline 28 & 37 & + & + & + & + & $\mathrm{M}$ \\
\hline 29 & 36 & - & + & + & + & $\mathrm{U}(-)$ \\
\hline 30 & 27 & + & + & + & - & $\mathrm{U}(-)$ \\
\hline 31 & 25 & + & + & - & + & $\mathrm{U}(-)$ \\
\hline 32 & 23 & + & - & - & - & $\mathrm{U}(+)$ \\
\hline 33 & 22 & - & + & + & + & $\mathrm{U}(-)$ \\
\hline 34 & 21 & + & - & - & - & $\mathrm{U}(+)$ \\
\hline 35 & 20 & - & - & - & + & $\mathrm{U}(+)$ \\
\hline
\end{tabular}




\begin{tabular}{|r|r|r|r|r|r|l|}
\hline 36 & 19 & + & + & + & + & $\mathrm{M}$ \\
\hline 37 & 18 & - & - & + & + & \multicolumn{1}{l|}{$\mathrm{P}$} \\
\hline 38 & 17 & + & + & - & + & $\mathrm{U}(-)$ \\
\hline 39 & 16 & + & + & + & + & $\mathrm{M}$ \\
\hline 40 & 15 & + & + & - & + & $\mathrm{U}(+)$ \\
\hline 41 & 14 & + & + & + & + & $\mathrm{M}$ \\
\hline 42 & 13 & + & + & + & + & $\mathrm{M}$ \\
\hline 43 & 12 & - & - & + & - & $\mathrm{U}(+)$ \\
\hline 44 & 11 & - & + & + & + & $\mathrm{U}(-)$ \\
\hline 45 & 10 & + & + & + & + & $\mathrm{M}$ \\
\hline & & & & & & $\mathrm{T}=112$ \\
& Total & 28 & 30 & 26 & 28 & \\
& & & & & & \\
\hline
\end{tabular}

Tab.2 Nombre de bandes présentes chez les génotypes étudiés à partir du polymorphisme protéique des graines. +: Présence, -:Absence, M: monomorphique, P:Polymorphique,

$\mathrm{U}$ : bande unique.

\begin{tabular}{|c|c|c|c|c|c|}
\hline \multirow{2}{*}{ Génotypes } & \multirow{2}{*}{$\begin{array}{c}\text { Bande } \\
\text { monomorphe }\end{array}$} & Bande unique & Bande non-unique & \multirow{2}{*}{ total } & \multirow{2}{*}{ Polymorphisme(\%) } \\
\cline { 3 - 5 } & 12 & 11 & 5 & 28 & 57.14 \\
\hline 1 & 12 & 1 & 17 & 30 & 60.00 \\
\hline 2 & 12 & 5 & 9 & 26 & 53.84 \\
\hline 3 & 12 & 7 & 9 & 28 & 57.14 \\
\hline 4 & & & 9
\end{tabular}

Tableau n`3: Taux de polymorphisme protéique des grains de quatre génotypes de la variété melanopus de blé dur cultivée en Algérie.

Le Dendrogramme de l'analyse phylogénétique a fait ressortir 3 groupes (Fig.8) :

- le premier groupe comprend le génotype G1

- le deuxième groupe situé entre le G1 et G4, est représenté par les génotypes G2, G3 qui semblent indiquer une origine génétique très proche traduisant une mono phylétique.

- le troisième groupe est constitué du génotype G4.

Les G1et G4 appartiennent au même grand groupe mais s'éloignent l'un de l'autre par rapport aux paramètres longueur de l'épi et la chlorophylle totale- traduisant une para phylétique. 


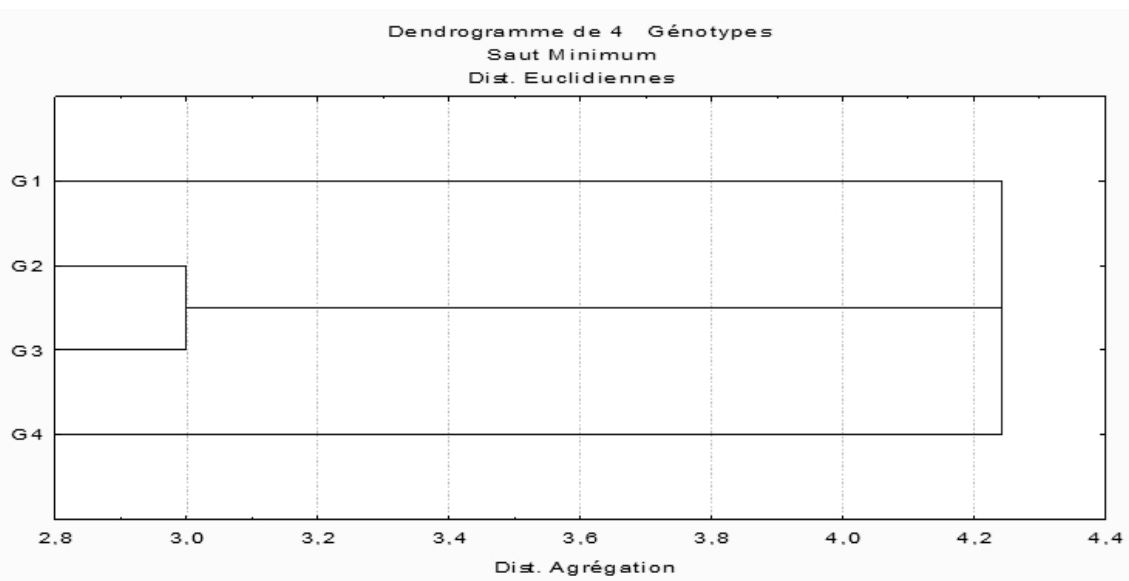

Fig.8. Dendrogramme d'après les profils électrophorétiques des graines des quatre génotypes de blé dur

Pour le blé dur, la teneur en protéines est une caractéristique essentielle considéré comme un critère important d'appréciation de la qualité. Les protéines représentent un intérêt technologique et nutritionnel pour l'utilisation du blé (Gate, 1995).

GERBA, 2013 a affirmé que la qualité du blé dur dépend de sa composition en protéines et que ce taux de protéines est conditionné par le génotype et influencé par l'environnement.

\section{Conclusion}

Les résultats des paramètres morpho-physiologiques et du rendement n'ont pas été concluants car ils n'ont pas tous montré de différence significative entre les quatre génotypes sauf pour les paramètres: surface foliaire(SF) et nombre d'épillets par épi (NE/E) qui ont permis de différencier les quatre génotypes.

En comparant les résultats des paramètres morpho-physiologiques et ceux des composantes du rendement, il ressort que les génotypes qui possèdent les valeurs les plus élevées pour les différents paramètres morpho-physiologiques sont ceux qui présentent les plus hauts rendements.

L'étude biochimique basée sur les protéines totales s'est révélée plus informative. Ces protéines ont permis de détecter un polymorphisme plus net entre les 4 génotypes, ainsi les génotypes $\mathrm{G} 2$ et G3 apparaissent très proches suggérant une monophylie alors que les génotypes G1 et G4 sont génétiquement éloignés.

\section{References:}

1. Albouchi, A., Sebei, H., Mezni, Y., \& EL Aouni M. H., (2000). Influence de la durée d'une alimentation hydrique déficiente 
sur la production de biomasse, la surface transpirante et la densité stomatique d'Acacia cyanophylla. Annales de l'INRGREF (4), p : 138- 61.

2. Amri, (2010). Diversité des gluténines HPM, sécalines HPM et 75 ysécalines d'une collection du CIMMYT de triticales hexaploides. Mémoire de magister, université Mentouri, Constantine. ; 69 p.

3. Anonyme 1 (2008). Centre régional de recherche et d'expérimentation en agriculture biologique Midi-Pyrénées résultats.

4. Anonyme 2 (2008). http://www.liberte-algerie.com

5. Auriau P., (1978). Sélection pour le rendement en fonction du climat chez le blé dur. Ann Argon d'El-Harrach. Vol 8 N², p : 1- 14.

6. Belkharchouche1, H., S., Fellah, H., Bouzerzour, A., Benmahammed et N.Chellal. (2009). vigueur de croissance, translocation et rendement en grains du blé dur (Triticum durum Desf.) sous condition semi-aride, $\mathrm{p}$ : 1-22.

7. Ben Mimoune, (1994). Caractérisation physiologique et organique de huit variétés de blé tendre en vue d'une sélection dans la zone sub humide .Thèse ING Blida, 54p.

8. Blottière, M.J., (2003). Cahier du centre de l'Algérie IX. Les productions algériennes. Chapitre Premier. Les productions agricoles.1. Les céréales. Publications du comité national métropolitain du centenaire de l'Algérie, Algérie, 95 p.

9. Boudour, L., (2006). Étude des ressources phyto-génétiques du blé dur (Triticum durum Desf.) algérien : analyse de la diversité génétique et des critères d'adaptation au milieu. Thèse Doctorat d'Etat. Université Mentouri Constantine, $142 \mathrm{p}$.

10. Chellali, B., (2007) Marché mondial des céréales : L'Algérie assure sa sécurité alimentaire.

11. http://www.lemaghrebdz.com/admin/folder01/une.pdf.

2007; (31.05.2008).

12. Erchidi, A.E., Benbella, M., et Talouizte, A., (2000). Relation entre certains paramètres contrôlant les pertes en eau et le rendement en grains chez neuf variétés de blé dur soumises au stress hydrique. Options méditerranéennes, série A (Séminaires Méditerranéens) (40), $\mathrm{p}: 279-82$.

13. Feillet, P., (2004). Valeur d'utilisation des blés durs .C.R. Sem. D’etudes Céréalicultures. Gembloux N (-85), p : 25-98.

14. Fischer R.A. ET Maurer R., (1978). Drought resistance in spring resistance wheat cultivar. I. Grain yieldresponses. Aust, J, Agri, Res, 29: 105-912.

15. Gate, P., 1995. Ecophysiologie du blé, de la plante à la culture. Lavoisier Tec and Doc. , Paris, $430 \mathrm{p}$. 
16. Gate P., Bouthier A., Casablanca H., et Deleens E., (1992). Caractères physiologiques décrivant la tolérance à la sécheresse des blés cultivés en France. Interprétation des corrélations entre le rendement et la composition isotopique du carbone des grains. In : Tolérance à la sécheresse des céréales en zone méditerranéenne. Diversité génétique et amélioration variétale. Montpellier (France) INRA. (Les colloques $\left.n^{\circ} 64\right), p: 61-74$.

17. Gerba, L., Getache, W., Bela, Y., Walelign W., (2013). Nitrogen Fertilization Effects on Grain Quality of Durum Wheat (Triticumturgidum L.Var.Durum) Varieties in Central Ethiopia. Journal of Agricultural Sciences Vol, 1(1), p: 1-7.

18. Hikosaka, K., Ishikawa, K., Borjigidai, A., Muller O. \& Onoda, Y., (2006). Temperature acclimation of photosynthesis: mechanisms involved in the changes in temperature Dependence of photosynthetic rate. J. Exp. Bot, p: 291 - 302.

19. Jasso, D., De Rodriguez., Romero-Garcia, J., Rodriguez-Garcia R. \& Sanchez J. L. (2002) Characterisation of proteins from sunflower leavesand seeds: Relationship of biomass and seed yield. Trends in new crops and new uses. ASHS Press. Alexandria. VA, p: 143-149.

20. Lebon, E., Pellegrino, A., Tardieu, F., \& Lecoeur, J., (2004). Shoot development in grapevine is

21. affected by the modular branching pattern of the stem and intra and inter-shoot trophic

22. competition. Annals of Botany, (93), p: 263 - 274.

23. Troccoli, A., Borrelli, G.G., De-Vita, P., Fares, C. et Di-Fonzoet, N., (2000). Mini review: durum wheat qualitty: a multidisciplinary concept. Jour. Of Cereal Science $\mathrm{N}^{\circ} 32$, p: $99-113$.

24. Mariana, (2011). Dynamique d'assemblage de protéines de réserve et du remplissage du grain de blé dur (Triticum durum Desf.).Docteur du center international D'études Supérieures en sciences Agronomique de Montpelier, p: 3 - 4.

25. Nemmar, M., (1980). Contribution à l'étude de la résistance à la sécheresse chez le blé tendre (Triticum aestivumL.) : Étude de l'accumulation de la proline sous l'effet du stress hydrique. Thèse D.A.A. ENSA. Montpellier, 65 p.

26. ONFAA, 2015. République Algérienne Démocratique et Populaire Ministère de l'Agriculture, du Développement Rural et de la Pêche Observatoire National des filières Agricoles et Agroalimentaires, $11 \mathrm{p}$.

27. Roudart, L. (2006). Terres cultivées et terres cultivables dans le monde. Paleohistoria $\mathrm{N}^{\circ} 48, \mathrm{p}: 150-156$. 
28. Slama, A., Ben Salem M., Ben Naceur, M., \& Zid E.D., (2005). Les céréales en Tunisie : production, effet de la sécheresse et mécanismes de résistance. Institut national de la recherche agronomiquede Tunisie (Inrat). Univ. Elmanar. Tunisie. (http://www.john-libbeyeurotext. fr/fr/revues/agro_biotech/sec/e-docs /00/04/11/2E/ telecharger.md), $\mathrm{p}: 225-229$. 